\title{
Seeing is believing - light for biomedical applications
}

\author{
Andrzej Kowalczyk ${ }^{*}$ \\ Faculty of Physics, Astronomy and Informatics, Nicolaus Copernicus University, Toruń, POLAND
}

Received December 22, 2011; accepted December 27, 2011; published December 31, 2011

\begin{abstract}
A brief overview is presented of the current issue of the Photonics Letters of Poland, devoted to biomedical applications of light.
\end{abstract}

Light is the most delicate probe to provide information on vulnerable structures in a noncontact and noninvasive way. It is used to sense biological tissues, art objects and molecular and atomic systems.

This issue contains invited papers which report research involving the application of light in the study of biological structures and processes.

Most papers are devoted to a well established technique of Optical Coherence Tomography (OCT).

The first paper from MIT, coauthored by James Fujimoto - the father of OCT, presents excellent visualization of microstructures of the anterior segment of the eye - especially its corneoscleral limbus.

The second contribution is from the laboratory of Rainer Leitgeb at the University of Vienna. It was professor Fercher - the former leader of this group who was a copioneer of OCT and invented its Fourier domain version.

Another example of structural imaging is the paper which presents combination of OCT with a cathether system to obtain images of the human esophagus. This paper was presented by Góra and Yoo from the laboratory of Bouma and Tearney in Massachusetts General Hospital.

Optical coherence tomography relies on light of high transversal but low longitudinal coherence. Biological research demands sources of various central wavelengths. This problem is addressed by the contribution from the group of Maciej Wojtkowski (University in Toruń)- a pioneer in applying spectral OCT to ophthalmology.

The optical system of the eye suffers from many aberrations. This makes images of the retina less resolved than those of the cornea. The solution is adaptive optics, which was introduced to OCT by R. Zawadzki from UC Davis.

\footnotetext{
*E-mail: akowal@fizyka.umk.pl
}

The problem of ocular aberrations is approached from the point of view of optometry by David Iskander from Wroclaw Technical University.

The internal structure of strongly scattering tissues is investigated by diffuse optical tomography. The contribution from A. Domański's group (Warsaw University of Technology) exploits the polarization of incoming and scattered photons to pinpoint structural elements hidden in the scattering medium.

Two contributions challenge blood dynamics. The joint effort of colleagues from Toruń and Wrocław provides some insight into the correlation between blood flow in the retina and movement of the apex of the cornea. The aim of this study is to provide a method to assess intraocular pressure.

An optical method to study blood aggregation and sedimentation, very useful in diagnostics, is presented by a group from Collegium Medicum of Nicolaus Copernicus University.

As an invited editor, I thank all contributors to this issue. I hope that these interesting articles will be read by the photonic community. 